\title{
Des instruments biographiques
}

In: Pôle Sud, N¹ - 1994. pp. 5-9.

Citer ce document / Cite this document :

Damamme Dominique. Des instruments biographiques. In: Pôle Sud, N¹ - 1994. pp. 5-9.

doi : 10.3406/pole.1994.1322

http://www.persee.fr/web/revues/home/prescript/article/pole_1262-1676_1994_num_1_1_1322 


\section{Des instruments biographiques}

\section{Dominique Damamme}

Programmant un sommaire centré sur la biographie, nous savions que nous ne ferions pas nécessairement œuvre pionnière, mais nous savions aussi que nous abordions une question vive : on en voudra pour preuves, tirées de l'actualité scientifique ou éditoriale récente, la traduction française en décembre 1993 de l'ouvrage de Nels Anderson, The Hobo (1923), le colloque sur " Les dictionnaires biographiques du mouvement ouvrier " qui s'est tenu en novembre dernier à Paris à l'initiative du CNRS ou celui de l'IEP de Rennes et la Revue Politix, "Les biographies en sciences sociales " (14-15 avril 1994). Si aujourd'hui la tentation biographique est aussi forte, au delà de l'attraction exercée par un marché de l'édition porté par l'attrait du public pour le "vécu " et le refus de la modernité romanesque, au delà encore des intérêts politiques très divers que peut servir la biographie (voir le culte des " grands hommes", ou la construction des identités politiques), c'est qu'elle se situe à la croisée de plusieurs tendances ou d'évolutions internes aux sciences sociales, la dévalorisation du marxisme, la promotion corrélative de l'individualisme et l'intérêt suscité par la sociologie compréhensive. Question vive, question ouverte aussi, les débats sur la méthode biographique ou le recours aux biographies ne risquant pas de se refermer de sitôt (J. Peneff, 1990). Croire et prétendre l'inverse serait s'aveugler sur l'irréductibilité des théories sociologiques et sur les possibilités de clore des interrogations méthodologiques qui sont d'abord affaire de théorie. Encore d'ailleurs faudrait-il savoir de quoi on parle dans la mesure où la méthode biographique n'en est pas une, qu'il ne s'agit pas d'une approche unique mais d'un pavillon couvrant plusieurs marchandises, autobiographie, biographie, biographie collective ou prosopographie, elles-mêmes inscrites dans des problématiques et des protocoles de recherche éminemment divers. Prudemment, dans cette brève note bibliographique, on se bornera, au sein d'un ensemble de recherches aussi vaste qu'hétérogène, à signaler quelques textes et quelques points saillants de la réflexion sur ces différentes démarches et sur leurs usages.

a) Mais auparavant, deux remarques liminaires : d'abord, de longue date, la sociologie a emprunté d'autres voies pour atteindre le programme le plus fort de la biographie, le plus "recommandable" pour reprendre les termes de J. C. Passeron (1990), qui consiste à ressaisir l'objectivation de la subjectivité et la subjectivisation de l'objectivité. Il suffit de citer L'éthique protestante et l'esprit du capitalisme. Ensuite, le choix scientifique reste ouvert entre une théorie sociologique et une théorie analytique (freudienne). Même si l'on donne à la 


\section{Pôle Sud}

notion d'habitus le sens large d'histoire sociale totale de "l'acteur " ou de "l'agent " - le choix du mot là non plus n'est pas neutre -, la tension subsiste entre ces deux principes d'analyse, comme on le voit en filigrane dans l'interprétation de L. Boltanski du Journal d'Amiel : "C'est dire, écrivait-il, que l'investissement intellectuel peut (...) tendre à éloigner des valeurs paternelles (...) c'est dire aussi que le surinvestissement sur la mère peut avoir pour effet de renforcer l'investissement intellectuel "(Bolstanski, 1975).

b) Le premier chantier qu'ouvre l'autobiographie (pour une définition, $P$. Lejeune) est celui, historique, de l'identification du genre, de ses commencements ou de ses recommencements. Il s'agit là du domaine immense de la sociogenèse de l'individu et de la personne (M. Mauss, $N$. Elias, L. Dumont, J. P Vernant...).

Le matériau autobiographique (individuel) - constitué d'écrits, le plus souvent mais non nécessairement, destinés à la publication et fréquemment associés à un désir de témoignage, d'auto-justification, voire d'édification - offre la possibilité d'étudier les représentations et les pratiques sociales et politiques, récurrentes ou plus ou moins singulières, d'un individu. C'est évidemment une large voie d'accès à l'analyse du personnel politique (E. Neveu). L'autobiographie, soit que l'individu présente un caractère modal ( $N$. Z. Davies), soit qu'à l'inverse il s'agisse d'un cas-limite (C. Ginzburg), peut encore être tenue comme un révélateur, comme un point de réfraction d'un groupe ( $G$. Duby, $D$. Roche), voire d'un univers culturel ( $E$.
Panofsky), sans qu'on puisse l'ériger en une incarnation d'une Zeitgeist au sens de Dilthey ou du système social global ( $F$. Ferrarroti).

Les avertissements sur les illusions qu'enferme l'autobiographie et qu'elle produit (P. Bourdieu, 1986; JC Passeron, 1990) sont suffisamment fondés pour qu'on se garde de les méconnaître et, notamment qu'on néglige le travail de rationalisation rétrospective du vécu social et de présentation de soi de la personne. Mais légitimité contre illégitimité, on n'oubliera pas non plus que $M$. Weber a façonné à partir de matériaux autobiographiques quelques-uns de ses types-idéaux (voir dans L'Éthique protestante et l'esprit du capitalisme son usage des écrits de B. Franklin). En fait, la pertinence du matériau est fonction de son traitement.

Enfin, puisque le chercheur est toujours inclus dans la construction de l'objet de la recherche, l'autobiographie, à titre d'autoanalyse, constitue un exercice salutaire bien qu'inusité. La tentative de G. Mauger et C. F. Poliak (1985) témoigne de l'intérêt que revêt un tel travail d'objectivation du lieu de l'objectivation, montrant les relations entre position sociale, investissements politiques et choix d'objets et de problématiques.

c) Originellement, la méthode biographique a eu partie liée avec une visée réformatrice ou critique : faire entendre la voix des dominés, de ceux qui ne prennent pas la parole ou à qui on l'a prise (J. Peneff).

S'interrogeant sur l'étude, au moyen de biographies, des " structures longitudinales de l'individualisation ", J. C Passeron distingue trois modalités principales. Une 


\section{Des instruments biographiques}

première forme de biographie, qualifiée d'analyse des " flux ", retrace l'inscription d'un itinéraire individuel dans l'ensemble des institutions sociales. Mais les contraintes sociales qui s'imposent comme modelages du comportement étant également le produit ou la résultante, par agrégation et incorporation, d'actions, s'oppose à ces biographies " en amont " une sociologie " en aval ", analyses des " carrières " et des " trajectoires". "Le projet le plus recommandable, écrit J. C Passeron, consiste à essayer de saisir la structuration des biographies à la fois comme un effet des structurations longitudinales qui se résument en amont dans "l'institution biographique" et comme le produit agrégé que l'action sociale des individus inscrit, en aval, dans le maintien ou la transformation de ces structures longitudinales ". L'analyse en termes de " carrière " renvoie à l'interactionnisme symbolique, notamment aux travaux d'H. Becker (1985) ou d'A Strauss. La seconde catégorie, l'analyse de " trajectoires ", s'attache à l'actualisation d'un habitus au travers des conjonctures qu'il traverse (P. Bourdieu, 1984). Ces projets, l'un comme l'autre, incluent le moment de la construction subjective, mais se séparent sur l'appréciation de l'autonomie de l'" opérateur pratique" (P. Bourdieu, 1987, 24) et sur le jeu de la conscience réflexive dans les " choix " individuels. On connaît la définition de P. Bourdieu de l'habitus comme " nécessité incorporée "ou "structure structurée structurante" $(1979,190)$ sans que "l'agent social " soit réduit, comme dans un certain marxisme structuraliste, à n'être plus que le porteur (Trager) de structures.
C'est très exactement cette question qui fonde la réflexion proposée par G. Levi (1989) sur les usages de la biographie. Ses distinctions ne recouvrent pas la classification de J. C Passeron : prosopographie, biographie " exemplaire "d'une époque, la biographie cas-limite, herméneutisme et biographie. Mais l'important n'est pas là. Cet exercice taxinomique est surtout pour lui une manière commode de poser la question de l'indétermination sociale, et cela à un double niveau : celui des incohérences entre normes sociales, et la nécessité de prendre en compte les espaces " interstitiels " propres à toute société ; celui, ensuite, de la tendance à présupposer la solidification des collectifs et, de cette solidité supposée, à conclure à l'homogénéité des habitus et à l'identité (problématique selon G. Levi) entre représentations collectives et représentations individuelles. Ainsi, le débat tourne autour des définitions et des usages de la notion d'habitus.

d) L'analyse prosopographique a servi d'abord à remédier à l'absence de renseignements précis ou homogènes sur une population, démultipliant ainsi les chances de saisie d'un groupe en " collectivisant " les données. En même temps, comme le rappelle C. Charle (1993), elle permet de travailler sur un collectif sans être prisonnier de sources sérielles et quantifiables préconstruites. En ce sens, la démarche présente l'avantage d'échapper au substantialisme en définissant les groupes à partir de leurs propriétés relationnelles. Méthode pour des micro populations, "avantgardes" de toute nature, artistique, intellectuelle ou politique, mais aussi pour des collectifs plus nombreux, la prosopographie 


\section{Pôle Sud}

renseigne sur la position, dominante ou dominée, d'un groupe dans l'espace social ou dans un champ social particulier. C'est cette visée qu'on retrouve dans les nombreuses biographies collectives de groupes sociaux comme les patronats ou encore les élus, ou de professions particulières comme les professeurs d'université, les instituteurs ou les médecins. Dans ses formes les plus abouties, la biographie sociale collective d'une catégorie particulière d'agents sociaux met à jour l'articulation entre, d'un côté, position sociale et identité, représentations et pratiques de l'autre, comme B. Pudal (1989) a pu le montrer dans son étude des cadres du PCF.

\section{Bibliographie}

a)

- P. Braud, Le jardin des délices démocratiques, Presses de la Fondation Nationale des sciences politiques, 1991.

- L. Bolstanski, Pouvoir et impuissance : projet intellectuel et sexualité dans le Joumal d'Amiel, Actes de la recherche en sciences sociales, 5/6, 1975, p 82.

- M. Catatni, S. Mazé, Tante Suzanne, Une histoire de vie sociale, Méridiens, 1982.

- E. Erikson, Luther avant Luther, Flammarion, 1968.

- Id. Gandi's Truth, New York, Norton, 1969.

- S. Freud, W Bullitt, Le président T.W. Wilson, Payot, 1990.

- R Hoggart, 33 Newport Street Gallimard, Le Seuil, 1991.

-V de Gaulejac : La névrose de classe, Hommes et Groupes Éditeurs, 1987.

- J. Laplanche, Holderlin et la question du pere, PUF, 1961.

- S. Miceli, Les intellectuels et le pouvoir au Brésil (1920-1945), PUG/MSH,1981.

- J. P. Sartre, L'idiot de le famille, Gallimard, 1971.

- J. Starobinski, J. J. Rousseau, La transparence et l'obstacle, Gallimard, 1971.

- M. Verret, Biographies, militances, dictionnaires, Colloque Les dictionnaires biographiques du mouvement ouvrier : lectures, exploitations, apports à l'historiographie, novembre 1993, CNRS.

- B. Vernier, La genese sociale des sentiments, aînés et cadets dans l'âle grecque de Karpathos, EHESS, 1991.

- P. Veyne, Comment on écrit l'histoire, Seuil, 1979.

b)

- H. Becker, Outsiders, Paris, Métailié, 1985.

- D. Bertaux, Histoire de vie ou récits de pratiques? Methodologie de l'approche biographique en sociologie, Paris, Rapport CORDES,1971.

- Id. L'approche biographique. Sa validité méthodologique, ses potentialités, Cahiers internationaux de sociologie, 2, 1980.

- L. Boltanski, Les cadres, Éditions de Minuit, 1982.

- P. Bourdieu, Esquisse d'une théorie de la pratique, Droz, 1979.

- Id. L'illusion biographique, Actes de la recherche en sciences sociales, 62-63, 1986.

- Id. Homo Academicus, Minuit, 1984.

- Id. Choses dites, Minuit, 1987.

- E. Durkheim, M Mauss, De quelques formes primitives de classification, in M. Mauss, Essais de sociologie, Editions de Minuit, 1968.

- L. Dumont, Essais sur l'individualisme, Seuil, 1983.

- N Elias, La dynamique de l'Occident, Flammarion. 


\section{Des instruments biographiques}

- F Ferraroti, Sur l'autonomie de la méthode biographique, in J. Duvignaud, Sociologie de la connaissance, 1979.

- Id. Histoire et histoires de vie, Méridiens, 1982.

- N. Heinich, Etre écrivain, Centre National des Lettres, 1990.

- P. Lejeune, L'autobiographie en France, A Colin, 1971.

- Id. Le pacte autobiographique, Seuil, 1975.

- G. Levi, Les usages de la biographie, Annales ESC, n 6, 1989.

- G. Mauger et C. F. Poliak, "Choix " politiques et " choix" de recherches : essai d'auto-socio-analyse (19731984), Jeunesses et sociétés, 1985.

- M. Mauss, Une catégorie de l'esprit humain : la notion de personne, celle de " moi ", in Sociologie et anthropologie, PUF.

- Momigliano, Les origines de la biographie en Grèce ancienne, Paris, Circé, 1992.

- E Panofsky, L'Abbé Surger de Saint Denis, in Architecture gothique et pensée scolastique, Minuit, 1967.

- A. Strauss, Miroirs et masques, Paris, Métailié, 1992.

- J. P Vernant, L'individu, la mort, l'amour, Soi-même et l'autre en Grèce ancienne, Gallimard, 1989.

c)

- N Anderson, The Hobo, Chicago, University Press, 1923.

- P. Berger, T Luckmann, La construction sociale de la réalite, Méridiens, 1986.

- P. Bourdieu, Homo Academicus, Paris, Ed de Minuit, 1984.

- id. Les règles de l'art, Paris, Seuil, 1992.

- A. Collovald, Identité (s) stratégique (s), Actes de la recherche en sciences sociales, n73, 1988.

- N. Z. Davies, AHR Forum : The return of Martin Guerre. On the lame, American Historical Review, $\mathrm{n}^{\circ}$ 93, 1988.

- J. M. Donegani, La liberté de choisir, Presses de la Fondation Nationale des Sciences Politiques, 1993.

- G. Duby, Guillaume le Maréchal, ou le meilleur chevalier du monde, Fayard, 1985.

- C. Ginzburg, Le fromage et les vers : l'univers d'un meunier du XVT siècle, Paris, Flammarion, 1988.

- G. Levi, Les usages de la biographie, Annales ESC, 6, 1989.

- E. Neveu, L'évolution des représentations indigènes du jeu politique à travers les autobiographies, Colloque Association Française de science politique, 1992.

- J. C. Passeron, Biographies, flux, itinéraires, trajectoires, Revue Française de Sociologie, 1, 1990.

- J. Peneff, La méthode biographique. De l'École de Chicago à l'histoire orale, Paris, A. Colin, 1990.

- D Roche, Journal de ma vie, Jacques-Louis Ménétra, compagnon vitrier au XVIIr siècle, Paris, Montalba, 1982.

- W. Thomas, F Znaniecki, The polish peasant in Europe and America, New-York, 1918-1920.

- A. Touraine, La voix et le regard, Seuil, 1978.

- M. Vovelle, L'Irrésistible Ascension de Joseph Sec, bourgeois d'Aix, Edisud, 1975.

- Id. Théodore Desorgues ou la désorganisation, Paris, Seuil, 1985.

- Id. De la biographie à l'étude de cas, in Actes du colloque "Problèmes et méthodes de la biographie ", 1985, Sources et travaux historiques, $\mathrm{n}^{\circ} 3 / 4,1985$.

d)

- C. Charle, J. Nagle, D Woronoff, Prosopographie des elites en France, XVT-XX, guide recherche, Paris, IHMC, 1980.

- C. Charle, Les élites de la République, Fayard, 1987.

- Id. L'invention des intellectuels, Minuit, 1990.

- Id. Du bon usage de la biographie sociale comparée ou les trois âges de la biographie collective, Colloque.

"Les dictionnaires biographiques du mouvement ouvrier", novembre1993.

- M. Dressen, Les étudiants à l'usine, Thèse IEP, 1992.

- B. Pudal, Prendre Parti, Presses de la Fondation Nationale des Sciences Politiques, 1989. 